\title{
Proton pump inhibitors and the risk of hospital-acquired acute kidney injury in children
}

\author{
Yanqin Li ${ }^{1}$, Mengqi Xiong ${ }^{1}$, Minliang Yang ${ }^{1}$, Long Wang ${ }^{1}$, Sheng Nie ${ }^{1}$, Diankun Liu ${ }^{1}$, Mingjing Pi ${ }^{1}$,
} Aihua Zhang ${ }^{2}$, Jianhua Mao ${ }^{3}$, Hai-Peng Liu ${ }^{4}$, Huimin $\mathrm{Xia}^{5}$, Hong Xu ${ }^{6}$, Zhangsuo Liu ${ }^{7}$, Shipin Feng ${ }^{8}$, Wei Zhou', Xuemei Liu ${ }^{10}$, Yonghong Yang ${ }^{11}$, Yuhong Tao ${ }^{12}$, Yunlin Feng ${ }^{13}$, Chunbo Chen ${ }^{14}$, Mo Wang ${ }^{15}$, Yan $\mathrm{Zha}^{16}$, Jian-Hua Feng ${ }^{17}$, Qingchu $\mathrm{Li}^{18}$, Shuwang Ge ${ }^{19}$, Jianghua Chen ${ }^{20}$, Yongcheng $\mathrm{He}^{21}$, Siyuan Teng ${ }^{22}$, Chuanming $\mathrm{Hao}^{23}$, Bi-Cheng Liu ${ }^{24}$, Ying Tang ${ }^{25}$, Wenjuan $\mathrm{He}^{1}$, Fan Fan Hou ${ }^{1 \#}$, Xin $\mathrm{Xu}^{1 \#}$

${ }^{1}$ National Clinical Research Center for Kidney Disease, State Key Laboratory of Organ Failure Research, Nanfang Hospital, Southern Medical University, Guangzhou, China; ${ }^{2}$ Children's Hospital of Nanjing Medical University, Nanjing, China; ${ }^{3}$ Children Hospital of Zhejiang University, Hangzhou, China; ${ }^{4}$ Anhui Institute of Pediatric Research, Anhui Provincial Children's Hospital, Hefei, China; ${ }^{5}$ Guangzhou Women and Children's Medical Center, Guangzhou Medical University, Guangzhou, China; ${ }^{6}$ Children's Hospital of Fudan University, Shanghai, China; ${ }^{7}$ The First Affiliated Hospital of Zhengzhou University, Zhengzhou, China; ${ }^{8}$ Chengdu Women and Children's Central Hospital, Chengdu, China; ${ }^{9}$ Shanghai Children's Medical Center, Shanghai Jiao Tong University, Shanghai, China; ${ }^{10}$ Jinan Children's Hospital, Jinan, China; ${ }^{11}$ Pediatric Medical Research Center, Gansu Province Child's Hospital, Lanzhou University Second Hospital, Lanzhou, China; ${ }^{12}$ West China Second University Hospital, Sichuan University, Chengdu, China; ${ }^{13}$ Sichuan Provincial People's Hospital, University of Electronic Science and Technology of China, Chengdu, China; ${ }^{14}$ Guangdong General Hospital, Guangdong Academy of Medical Sciences, Guangzhou, China; ${ }^{15}$ Children's Hospital of Chongqing Medical University, Chongqing, China; ${ }^{16}$ Guizhou Provincial People's Hospital, Guizhou University, Guiyang, China; ${ }^{17}$ The Second Affiliated Hospital, Zhejiang University, Hangzhou, China; ${ }^{18}$ Guilin Medical University Affiliated Hospital, Guilin, China; ${ }^{19}$ Tongji Hospital Affiliated to Tongji Medical College, Huazhong University of Science and Technology, Wuhan, China; ${ }^{20}$ Kidney Disease Center, the First Affiliated Hospital, Zhejiang University, Hangzhou, China; ${ }^{21}$ Center for Nephrology and Urology Shenzhen University, the First Affiliated Hospital of Shenzhen University, Shenzhen University, Shenzhen, China; ${ }^{22}$ The Second Affiliated Hospital of Dalian Medical University, Dalian, China; ${ }^{23}$ Huashan Hospital, Fudan University, Shanghai, China; ${ }^{24}$ Institute of Nephrology, Zhong Da Hospital, Nanjing, China; ${ }^{25}$ Sun Yat-sen Memorial Hospital, Sun Yat-sen University, Guangzhou, China

Contributions: (I) Conception and design: X Xu, FF Hou; (II) Administrative support: X Xu, FF Hou, S Nie; (III) Provision of study materials or patients: D Liu, M Pi, A Zhang, J Mao, HP Liu, H Xia, H Xu, Z Liu, S Feng, W Zhou, X Liu, Y Yang, Y Tao, Y Feng, C Chen, M Wang, Y Zha, JH Feng, Q Li, S Ge, J Chen, Y He, S Teng, C Hao, BC Liu, Y Tang, W He; (IV) Collection and assembly of data: L Wang, M Xiong; (V) Data analysis and interpretation: Y Li, S Nie, M Yang; (VI) Manuscript writing: All authors; (VII) Final approval of manuscript: All authors.

\#These authors contributed equally to this work.

Correspondence to: Dr. Xin Xu; Dr. Fan Fan Hou. Renal Division, Nanfang Hospital, Southern Medical University, 1838 North Guangzhou Ave, Guangzhou 510515, China. Email: xux007@163.com; ffhouguangzhou@163.com.

Background: To evaluate the association between use of proton pump inhibitor (PPI) and the risk of hospital-acquired acute kidney injury (HA-AKI) in hospitalized children.

Methods: We conducted a multicenter retrospective cohort study in hospitalized children aged 1 month to 18 years from 25 tertiary hospitals across China from 2013 to 2015. Patient-level data were obtained from the electronic hospitalization databases. AKI was defined and staged using the serum creatinine (SCr) data according to the Kidney Disease Improving Global Outcomes (KDIGO) criteria.

Results: Among 42,232 children analyzed, 11,496 (27.2\%) used PPI, 1,760 (4.2\%) used histamine 2 receptor antagonist (H2RA), and 3,514 (8.3\%) had HA-AKI during hospitalization. Over $85 \%$ of PPIs were prescribed for prophylaxis of gastro-duodenal lesions in children. The use of PPI was associated with a significantly increased risk of HA-AKI compared with both non-users [odds ratio (OR), 1.37; 95\% confidence interval (CI), 1.23-1.53)] and H2RA users (OR, 1.24; 95\% CI, 1.01-1.52). The associations were consistent across children of different age range, gender, subtypes of PPIs and methods of administration. A larger effect was observed in children with chronic kidney disease (OR, 3.37; 95\% CI, 2.46-4.62) and those 
needed intensive care (OR, 1.54; 95\% CI, 1.33-1.78). The risk of HA-AKI was increased even within the recommended dosage range of PPI.

Conclusions: PPIs were widely used and associated with an increased risk of HA-AKI in hospitalized children in China.

Keywords: Proton pump inhibitors (PPIs); hospital-acquired acute kidney injury (HA-AKI); pediatrics; nephrotoxicity

Submitted Mar 07, 2020. Accepted for publication Aug 21, 2020.

doi: $10.21037 / \mathrm{atm}-20-2284$

View this article at: http://dx.doi.org/10.21037/atm-20-2284

\section{Introduction}

Acute kidney injury (AKI) is a rapidly growing public health challenge, with incidences ranging from $11.6 \%$ to $18.3 \%$ in adults and $19.6 \%$ to $26.9 \%$ in children during hospitalization (1-6). AKI is associated with a substantially increased risk of death and other adverse outcomes (6-8). Children with AKI may have long-term sequelae that lead to chronic kidney disease (CKD) and permanent loss of kidney function in their adult life (9-12). Exposure to nephrotoxic medications has been recognized as a common cause of hospital-acquired (HA) AKI, contributing to $16-28 \%$ of the AKI risk in children $(13,14)$. Avoiding inappropriate use of nephrotoxic drugs is pivotal for preventing children from developing iatrogenic HA-AKI.

Acid-suppressants, mostly proton pump inhibitor (PPI) and histamine 2 receptor antagonist (H2RA), are among the most widely prescribed medications worldwide. They are commonly prescribed for treatment of gastrointestinal disorders, such as gastroesophageal reflux disease (GERD), gastro-duodenal ulcers, erosive esophagitis, helicobacter pylori gastritis (15-17), and for prophylaxis of gastroduodenal lesions. Notably, use of acid-suppressants in children surged substantially during last decades, which could not be commensurate with the prevalence of GERD $(18,19)$. Forgacs et al. estimated that $25-70 \%$ of PPIs were prescribed without appropriate indications (20-22). At present, high-quality evidence of benefit of PPIs in infants and children is lacking.

The use of PPI has been linked to increased risk of acute interstitial nephritis (AIN) and AKI in adults (23-26). A meta-analysis including over 2 million adult patients suggested that PPI users had a $44 \%$ higher risk of AKI than non-users (27). However, data on the use of PPI and its association with the risk of AKI in pediatric patients was non-existent.
We have previously established a large multicenter cohort including 3,044,244 hospitalized patients admitted from 2013 to 2015 across China (Epidemiology of AKI in Chinese Hospitalized patients, EACH study) $(3,14,28)$. Recently, we have found that the use of PPI was also associated with an increased risk of pediatric AKI (14), although the analysis was only limited to the absence or presence of the drug exposure. In the current study, we aim to provide a comprehensive analysis on the association between the use of PPI and the risk of HAAKI in hospitalized children, including possible effect modification by dose, subtypes, and administration methods of PPIs, comorbidities and other concomitant medications.

We present the following article in accordance with the STROBE reporting checklist (available at http://dx.doi. org/10.21037/atm-20-2284).

\section{Methods}

\section{Study design, population and data source}

$\mathrm{EACH}$ is a large-scale, multicenter, retrospective cohort study. We invited 32 tertiary academic medical centers in 16 provinces across China to participate in the study. A total of 25 centers, including 9 children's hospitals and 16 general hospitals that spanned 15 provinces, agreed. The cohort included 948,495 pediatric inpatients aged between 1 month and 18 years who were admitted from January 1, 2013 to December 31, 2015. We selected the pediatric patients who had at least two serum creatinine (SCr) tests within any 7-day window during their first 30 days of hospitalization and excluded those (I) diagnosed as end stage renal disease, or receiving maintenance dialysis or renal transplantation; (II) diagnosed as community acquired AKI (patients who met at least one of 


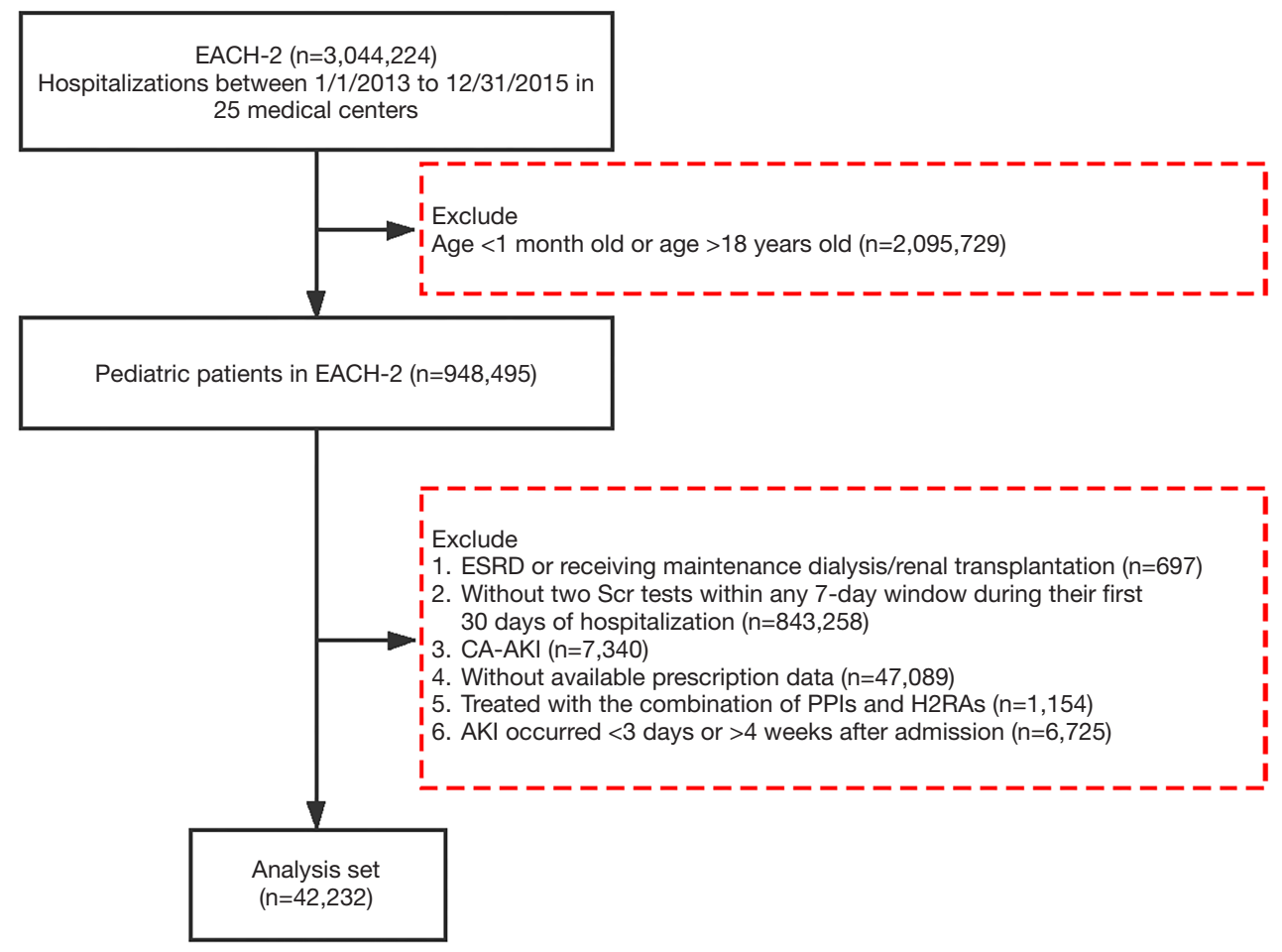

Figure 1 Flowchart of patient selection.

the following criteria were classified as having communityacquired AKI $(3,14,28)$ : (i) admission diagnosis code contains AKI; (ii) SCr change on the first day of admission met the Kidney Disease Improving Global Outcomes (KDIGO) definition); (III) missing prescription data; (IV) AKI occurred within the first 3 days or after 4 weeks of admission, and $(\mathrm{V})$ with combination therapy of both PPI and H2RA (Figure 1). For patients with multiple hospitalizations, we only included the first hospitalization.

Patient-level data were obtained from the electronic hospitalization databases, laboratory databases, and prescription databases from the participating centers. The hospitalization records consisted of patients' age, gender, residence, date of birth and diagnosis code at admission and discharge, operation procedures and dates, need for intensive care. The laboratory data included value and time of patients' SCr tests. The prescription data included the drug or procedures prescribed, dose, method of administration, frequency, and start and stop time. The exported data from all study centers were pooled and cleaned at the National Clinical Research Center for Kidney Disease in Guangzhou. The study was conducted in accordance with the Declaration of Helsinki (as revised in 2013). The study was approved by The Medical Ethics Committee of Nanfang Hospital (No. NFEC-2014-098) and individual consent for this retrospective analysis was waived.

\section{Identification of $\mathrm{HA}-\mathrm{AKI}$}

AKI was defined as an increase in SCr by $\geq 26.5 \mu \mathrm{mol} / \mathrm{L}$ $(0.3 \mathrm{mg} / \mathrm{dL})$ within 48 hours or $\mathrm{a} \geq 50 \%$ increase of $\mathrm{SCr}$ within 7 days according to the KDIGO criteria (29). Patients who had at least two SCr tests within a 7-day window were screened for AKI using an algorithm described previously $(3,14,28)$. Briefly, at any time point denoted by $t$, a baseline creatinine was dynamically defined as the mean creatinine level within the last 90 days before $t$, and each of the available creatinine data within 7 days after $t$ was compared with the baseline. The earliest day that the creatinine change met the KDIGO criteria was defined as the date of AKI detection.

The stage of AKI was determined using the peak SCr level after AKI detection, with increase of $<100 \%, 100$ $199 \%$ and $\geq 200 \%$ over the baseline being defined as stage 1 , 2 and 3 , respectively. 


\section{Definition of drug use}

Drugs were classified according to the Anatomical Therapeutic Chemical (ATC) classification system. The prescribed PPIs included omeprazole (A02BC01), pantoprazole (A02BC02), lansoprazole (A02BC03), rabeprazole (A02BC04), and esomeprazole (A02BC05). The prescribed H2RAs included cimetidine (A02BA01), ranitidine (A02BA02), famotidine (A02BA03), and roxatidine (A02BA06). Drug use was defined as any filled prescription of the specified drug within 2 weeks prior to the detection date of AKI in patients with HA-AKI and within 2 weeks prior to the second SCr test date in the 7-day test window in those without AKI. Drug exposure was also quantified using the cumulative dose of drug filled within the 2-week period standardized by body weight. Concomitant use of other nephrotoxic drugs, including non-steroid anti-inflammatory drugs (NSAIDs), chemotherapy agents, contrast medium, corticosteroids, angiotensin converting enzyme inhibitors (ACEIs), angiotensin receptor blocker (ARB), thiazide diuretic, loop diuretic and nephrotoxic antibiotics (Table S1) were determined similarly.

\section{Outcome and clinical comorbidities}

The primary outcome of this study was HA-AKI. Presence of comorbidities, including glomerulonephritis, hypertension, diabetes mellitus, congenital heart diseases, hematological malignancy, pneumonia, asthma, diarrhea, epilepsy, heart failure, malnutrition, sepsis, shock, systemic lupus erythematosus, trauma, tuberculosis, hepatitis, urinary tract obstruction, burn, low birth weight, and acid-suppression related gastrointestinal diseases, was determined by the International Classification of Diseases, tenth Revision (ICD-10) diagnosis codes on admission and at discharge. Acid-related gastrointestinal diseases included gastroesophageal reflux disease, gastrointestinal bleeding, ulcer disease, Helicobacter pylori infection, Barrett esophagus and achalasia. The diagnosis codes of clinical comorbidities were listed in the Table S2.

\section{Statistical analysis}

Children treated with the combination of PPI and H2RA were excluded from the study, and the rest were classified into PPI users, H2RA users and non-users accordingly. The baseline characteristics of the study population were summarized by strata of exposure to acid-suppressant. Continuous variables were presented as mean \pm standard deviation (SD) or median ( $25^{\text {th }}, 75^{\text {th }}$ quantile). Categorical variables were presented as counts and percentages. Comparisons between groups were performed using ANOVA tests or Kruskal-Wallis tests for continuous variables, and Chi-square tests for categorical variables.

Propensity scores for PPI use were calculated using a logistic regression model with predictors including age, gender, hospital and division of admission, baseline SCr, time from admission, comorbidities, operation procedures, need for intensive care and exposure to other nephrotoxic drugs. The effect of PPI use on the risk of HA-AKI was estimated using three different logistic regression models. Separate regression analyses were performed, with nonusers and H2RA users as the reference group, respectively. Model 1 adjusted for age and baseline SCr only. Model 2 adjusted for, in addition to those included in Model 1 , gender, hospital and division of admission, number of $\mathrm{SCr}$ tests within the 7-day window, maximum testing interval within the 7-day window, comorbidities, operation procedures, need for intensive care and the use of other nephrotoxic drugs. Model 3 included the same set of covariates as in Model 2 and weighted each observation by the inverse probability of treatment assignment, with the weight of $1 / p$ for PPI users and $1 /(1-p)$ for non-users, where $p$ is the propensity score (30). In the analysis, only patients with overlapped propensity scores between the treated and the non-treated were included. For better robustness, weights over 20 were truncated to 20 . In the regression models, natural spline terms $(\mathrm{df}=4)$ were used for the adjustment of quantitative variables such as age, baseline SCr and time from admission. Possible interactions of PPI use with age, gender, CKD, need for intensive care, operation and NSAIDs use were also examined using the regression model.

\section{Sensitivity analyses}

Four sensitivity analyses were performed. First, the effect of PPI use on the risk of HA-AKI was estimated in a 1:1 propensity score matched cohort (PPI users $v s$. non-users). Three different matching methods were considered, including (I) nearest-neighbor matching without replacement and with a caliper width of 0.001 , (II) nearest-neighbor matching without replacement and with a caliper width of $0.2^{*} \mathrm{SD}$ of the logit of the estimated propensity score, and (III) exact matching on age, sex, 
need for intensive care and received operation and nearestneighbor matching without replacement and with a caliper width of $0.2^{*} \mathrm{SD}$ of the logit of the estimated propensity score. The performance of these three matching methods were summarized in Tables S3-S5, respectively. The second matching method yielded the largest sample size $(n=8,123$ pairs) and a satisfactory maximal of absolute standardized mean of difference (0.031), and therefore was used for the subsequent sensitivity analysis. Second, the robustness of our main analysis was assessed by defining AKI according to a criterion based on the reference change value of $\mathrm{SCr}$ (pROCK) that had been shown to be more optimized than KDIGO in identifying pediatric AKI (28). Third, the analysis was restricted to "new" PPI user in order to reduce possible confounding effects by children who had been on PPI before admission. In the analysis, children who used PPI only after 5 days of admission (i.e., with at least a 5-day wash-out period) were defined as "new" PPI users. Finally, to account for competing risks of death and AKI, an analysis was performed on a composite outcome that included either AKI or death.

All statistical analyses were performed using $\mathrm{R}$ version 3.5.3 (31).

\section{Results}

\section{Study population}

Of 948,495 hospitalized children, 42,232 met our inclusion and exclusion criteria and were selected for further analyses (Figure 1). Among them, 11,496 (27.2\%) and 1,760 (4.2\%) were users of PPI and H2RA, respectively. The characteristics of the study population stratified by drug use were summarized in Table 1 . The characteristics of the included and the excluded children with prescription data were presented in Table S6. Compared with non-users and H2RA-users, PPI users were older, had a higher baseline SCr, and were more likely to receive intensive care and operation. The three groups differed significantly in exposure to other nephrotoxic drugs. Omeprazole constituted $67.5 \%$ of the PPI prescribed in children (Table S7). PPI use in various clinical settings was depicted in Figure S1. Notably, over $85 \%$ of PPIs were prescribed to children receiving operation, needing intensive care, or taking corticosteroids or non-steroidal anti-inflammatory drugs, presumably for prophylaxis of gastro-duodenal lesions (Table S8). Only $2.9 \%$ of PPI users had GERD.

\section{Use of PPI was associated with an increased risk of $H A-A K I$}

A total of 962 (8.4\%) and 2,390 (8.2\%) HA-AKI events were identified among PPI users and non-users, respectively. Compared with non-users, PPI users had $37 \%$ higher odds of HA-AKI (OR, 1.37; 95\% CI, 1.23-1.53) after adjusting for age, gender, hospital and division of admission, the number of and maximum time interval between SCr tests, baseline SCr, comorbidities, operation procedures, need for intensive care, and the use of other nephrotoxic drugs (Table 2). Similar sizes of effect were observed among different subtypes and administration methods of PPIs (Table S9). PPI use was also associated with a significantly increased risk of severe AKI defined as doubling of $\mathrm{SCr}$ or need for dialysis (Table S10) (32).

Compared with H2RA users who shared similar prescription indications, PPI users had 24\% higher odds of HA-AKI (OR, 1.24; 95\% CI, 1.01-1.52) after adjusting for the covariates (Table 2). Notably, H2RA might also be associated with an increased risk of HA-AKI in children. Compared with non-users, H2RA users had a 35\% higher risk of HA-AKI in our study (Table S11).

Children with CKD or needing intensive care had a higher risk of PPI-related AKI than those without CKD ( $\mathrm{P}$ for interaction $<0.001$ and 0.02 , respectively, Table 3). Age, gender, receiving operation, and concomitant use of NSAIDs did not significantly modify the effect of PPI use on HA-AKI.

\section{Adverse renal effect of PPI was observed within recommended dosage range}

The distribution of cumulative exposure days of PPI was presented in Figure S2. Since omeprazole was by far the most commonly used PPI during hospitalization, we estimated the distribution of the cumulative dose of omeprazole (Figure S3) and the dose-response curve of the cumulative dose of omeprazole and risk of HA-AKI in children (Figure 2). The risk of AKI was significantly increased even at a cumulative dosage of $1 \mathrm{mg} / \mathrm{kg}$ of body weight which was equivalent to 1 -day use at the lowest recommended daily dose. The risk of AKI peaked at around $6 \mathrm{mg} / \mathrm{kg}$ of body weight and remained flat after.

\section{The association between PPI use and the risk of $H A-A K I$ was robust in the sensitivity analyses}

In the propensity score-matched analysis, the effect of 
Table 1 Characteristics of hospitalized children stratified by use of PPI and H2RA

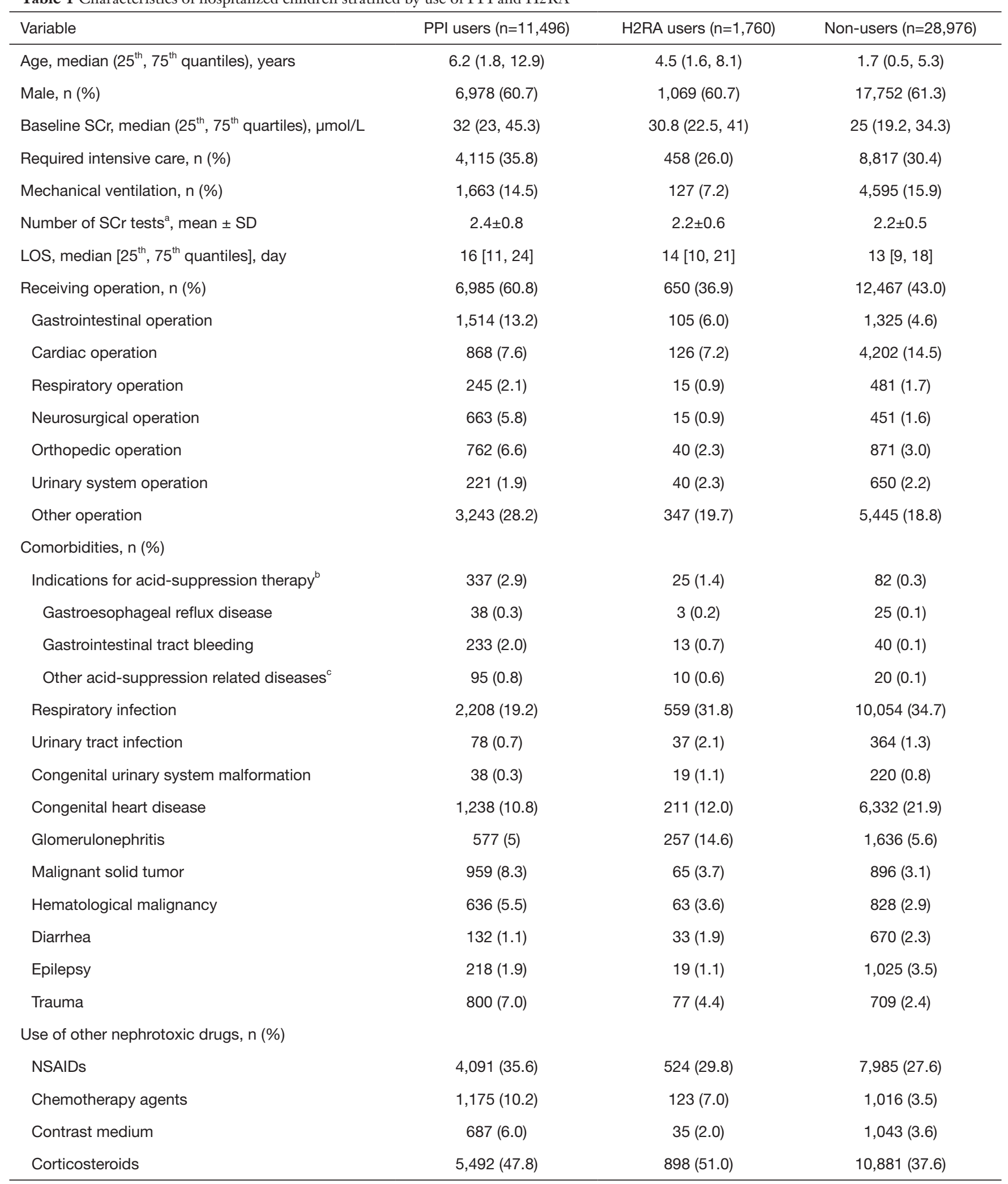

Table 1 (continued) 
Table 1 (continued)

\begin{tabular}{|c|c|c|c|}
\hline Variable & PPI users $(n=11,496)$ & H2RA users $(n=1,760)$ & Non-users $(n=28,976)$ \\
\hline ARB & $50(0.4)$ & $8(0.5)$ & $45(0.2)$ \\
\hline Thiazide diuretic & $528(4.6)$ & $147(8.4)$ & $2,076(7.2)$ \\
\hline Loop diuretic & $4,028(35)$ & $587(33.4)$ & $9,821(33.9)$ \\
\hline Number of nephrotoxic drugs ${ }^{\mathrm{e}}$, mean $\pm \mathrm{SD}$ & $1.9 \pm 1.4$ & $1.8 \pm 1.3$ & $1.6 \pm 1.4$ \\
\hline
\end{tabular}

${ }^{a}$, number of serum creatinine tests within the 7-day window; ${ }^{b}$, the indications might be overlapped; ${ }^{c}$, including peptic ulcer, $H$. pylori infection, Barrett esophagus, and achalasia; ${ }^{d}$, including aminoglycosides, vancomycins, sulfonamides, antifungals, antivirals, tetracycline, first-generation cephalosporins, semisynthetic penicillin and antituberculous drugs; ', average number of nephrotoxic medications used in each group. SCr, serum creatinine; SD, standard deviation; LOS, length of stay in hospital; PPI, proton pump inhibitor; H2RA, histamine 2 receptor antagonist; NSAIDs, non-steroid anti-inflammatory drugs; ACEl, angiotensin converting enzyme inhibitors; ARB, angiotensin receptor blocker.

Table 2 Use of PPI and the risk of HA-AKI in children

\begin{tabular}{|c|c|c|c|c|c|c|c|c|c|c|}
\hline Subgroup & $\begin{array}{c}\text { Total } \\
\text { patients, N }\end{array}$ & $\begin{array}{l}\text { HA-AKI } \\
\text { events, N }\end{array}$ & $\begin{array}{l}\text { Unadjusted } \\
\text { OR (95\% Cl) }\end{array}$ & $P$ value & \multicolumn{2}{|c|}{ Model $1^{\mathrm{a}}$} & \multicolumn{2}{|c|}{ Model $2^{b}$} & \multicolumn{2}{|c|}{ Model $3^{c}$} \\
\hline \multicolumn{11}{|c|}{ PPI users vs. non-users } \\
\hline Non-users & 28,976 & 2,390 & Reference & & Reference & & Reference & & Reference & \\
\hline \multicolumn{11}{|c|}{ PPI users vs. H2RA users } \\
\hline H2RA users & 1,760 & 162 & Reference & & Reference & & Reference & & Reference & \\
\hline PPI users & 11,496 & 962 & $\begin{array}{c}0.90 \\
(0.76-1.08)\end{array}$ & 0.24 & $\begin{array}{c}1.18 \\
(0.97-1.42)\end{array}$ & 0.09 & $\begin{array}{c}1.24 \\
(1.01-1.52)\end{array}$ & 0.04 & $\begin{array}{c}1.22 \\
(0.92-1.63)\end{array}$ & 0.17 \\
\hline
\end{tabular}

\footnotetext{
${ }^{\mathrm{a}}$, Model 1: adjusted for age, baseline SCr; ${ }^{\mathrm{b}}$, Model 2: adjusted for covariates in model 1 and gender, hospital, division of admission, number of serum creatinine tests within the 7-day window, maximum testing interval within the 7-day window, comorbidities, operation procedures, need for intensive care and use of other nephrotoxic drugs; ' , Model 3: adjusted for covariates in model 2 and weighted by inverse probability of treatment assignment. HA-AKI, hospital-acquired acute kidney injury; PPI, proton pump inhibitor; H2RA, histamine 2 receptor antagonist; OR, odds ratio; $\mathrm{Cl}$, confidence interval.
}

PPI use on the risk of HA-AKI was comparable to that in the main analysis with an adjusted OR of 1.36 (95\% CI, 1.20-1.55) (Table S12). The OR of PPI use was 1.56 (95\% CI, 1.34-1.80) while using a new definition optimized for pediatric AKI (pROCK), and 1.81 (95\% CI, 1.52-2.17) while comparing new PPI users to non-users (Tables S13,S14). The effect size of PPI use on the composite outcome including either HA-AKI or death was very similar to that of HA-AKI alone (Table S15).

\section{Discussion}

In this large cohort of hospitalized children in China, PPIs were prescribed to nearly $30 \%$ of children, mostly to children who needed intensive care, received operation, or took corticosteroids or NSAIDs, presumably for prophylaxis of gastro-duodenal lesions. Our study identified use of PPI as an independent risk factor for HA-AKI in children and the risk was significantly elevated even within 
Table 3 Association between PPI use and HA-AKI stratified by subgroups

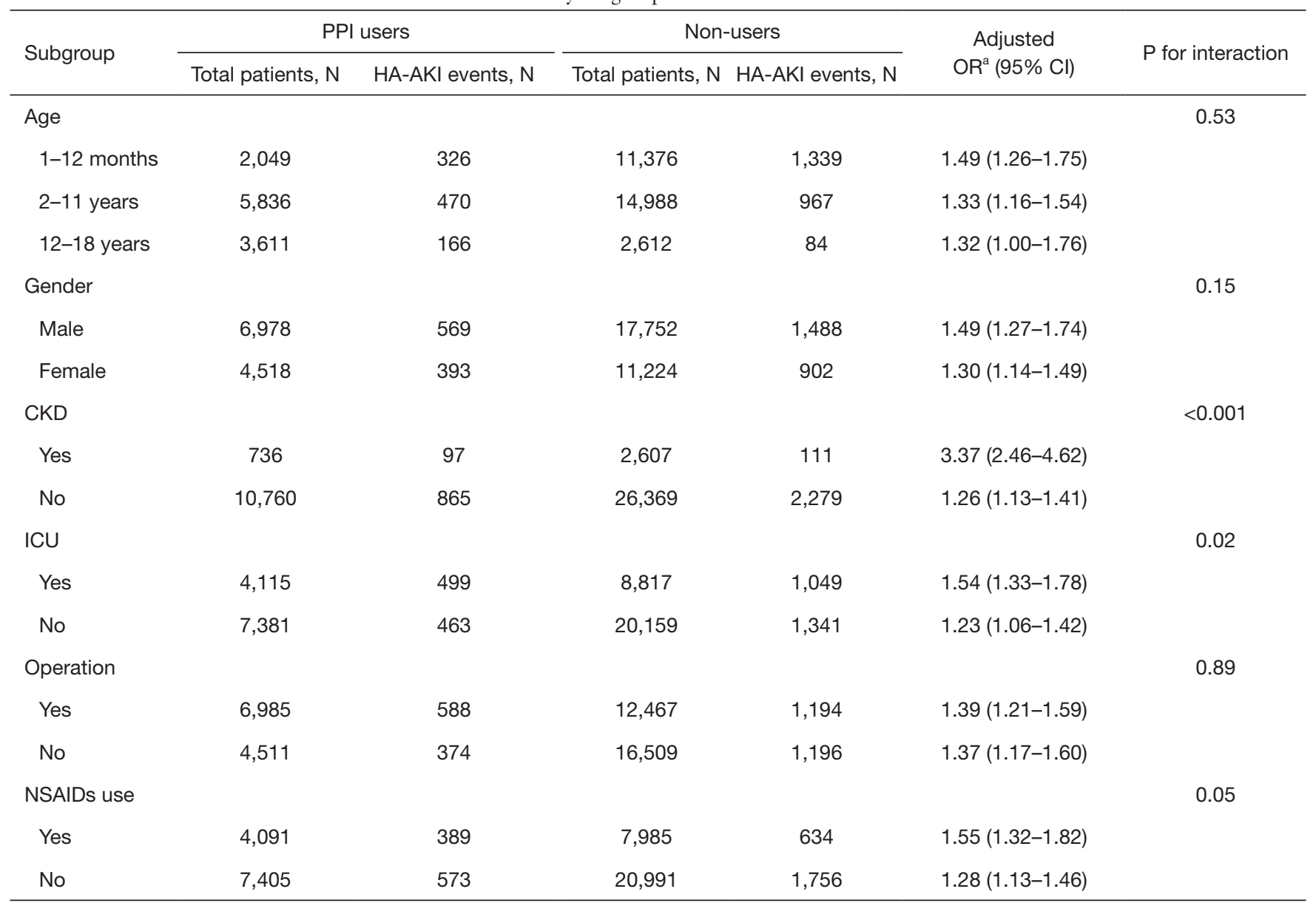

${ }^{a}$, adjusted for age, gender, hospital, division of admission, number of serum creatinine tests within the 7-day window, maximum testing interval within the 7-day window, baseline SCr, comorbidities, operation procedures, need for intensive care and use of other nephrotoxic drugs. PPI, proton pump inhibitor; CKD, chronic kidney disease; ICU, intensive care unit; NSAIDs, non-steroid anti-inflammatory drugs; $\mathrm{OR}$, odds ratio; $\mathrm{Cl}$, confidence interval.

the recommended dosage range of PPI. The effect of PPI appeared to be independent of drug subtypes, methods of administration, age range and gender of children. However, children with CKD or needing intensive care were more susceptible to PPI-related AKI than those without CKD. Our results called for judicious use of PPI and close monitoring of renal function in these high-risk population.

PPIs are commonly prescribed, available over-thecounter, and taken by millions of patients worldwide (20,33-37). Increasing trend of overutilization of acidsuppression medications in children was reported in recent studies $(18,38,39)$. Consistent with these reports, $27.2 \%$ of hospitalized children in our cohort used PPI, of whom only $3 \%$ were diagnosed with acid-related diseases. We speculated that most of the prescriptions were for ulcer prophylaxis since majority of the PPI users were children who needed intensive care, received operation, or took corticosteroids or NSAIDs. Previous studies have demonstrated that PPI use significantly reduces the risk of gastrointestinal bleeding in hospitalized patients (40). Our findings suggest that an individualized assessment of risk and benefit is needed when prescribing PPI for prophylaxis purpose, especially to patients who are at high risk of AKI.

The association between PPI use and AKI in adults has been well documented in previous studies $(23-27,41,42)$. In our pediatric cohort, the use of PPI was associated with a 37\% higher risk of HA-AKI. The effect size is similar to that observed in adults (hazard ratios, 1.30 to 1.54 ) (42). Our study showed that the adverse effect of PPI on the risk of AKI was consistent across children of different 


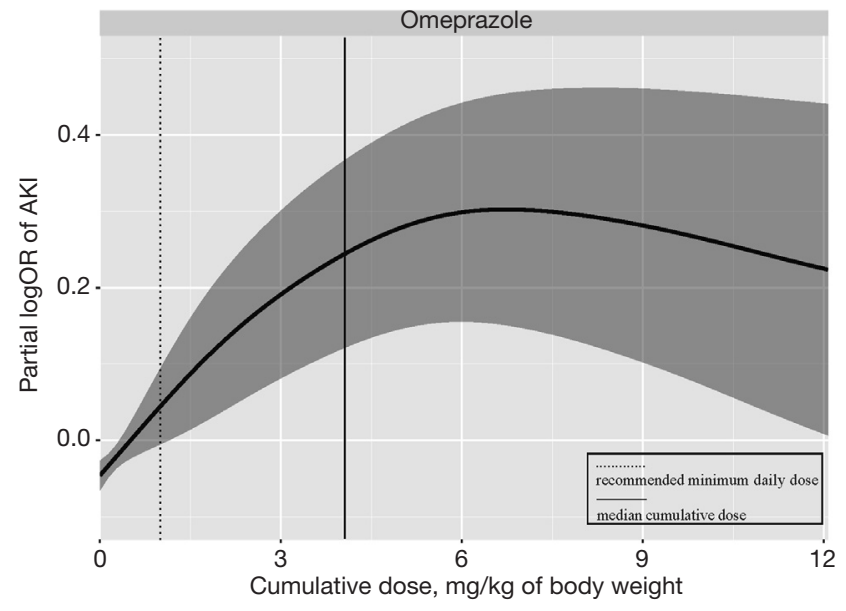

Figure 2 The dose response curve of omeprazole and the risk of HA-AKI. Odds ratio was adjusted for age, gender, baseline SCr, hospital and division of admission, number of serum creatinine tests within the 7-day window, maximum testing interval within the 7-day window, comorbidities, operation procedures, need for intensive care and the use of other nephrotoxic drugs. The dotted vertical line indicates the recommended minimum daily dose for omeprazole, the solid vertical line indicates the median cumulative dose of omeprazole. HA-AKI, hospital-acquired acute kidney injury; $\mathrm{SCr}$, serum creatinine.

age range and gender, subtypes of PPIs and methods of administration. As with any large observational study of a therapeutic intervention, the risk of confounding by indication is large and cannot be ignored. However, we believed that the increased risk of AKI was specific to the exposure of PPI in our study, not to the drug indications or other concomitant nephrotoxic medications, for the following reasons. First, in our main analysis, we have adjusted for various clinical settings, including CKD, operations, need for intensive care, acid-suppression related gastrointestinal diseases and concomitant use of other nephrotoxic drugs, including NSAIDs, chemotherapy agents, contrast medium, corticosteroids, angiotensin converting enzyme inhibitors, ARB, thiazide diuretic, loop diuretic and nephrotoxic antibiotics. Second, the association between PPI use and the risk of HA-AKI was consistently observed in all subgroups stratified by age, gender, CKD, need for intensive care, receiving operation and use of NSAIDs. Third, the association was robust in the four sensitivity analyses performed.

The mechanisms by which PPI induces AKI remain to be elucidated. AIN with mononuclear cells infiltration in the tubular interstitium has been reported in patients who develop PPI-related AKI $(43,44)$. Perazella et al. suggested that PPI use lead to endothelial dysfunction and increased oxidative stress, which might increase the risk of kidney damage (45). PPI has been shown to modulate the cellmediated immune response that might be idiosyncratic and dose-independent $(44,46,47)$. Since the recommended daily dose of omeprazole for gastroesophageal reflux in children is $1-4 \mathrm{mg} / \mathrm{kg} / \mathrm{d}$ (15), the effect of omeprazole on AKI was significantly increased after only 1 day of use in our observation, suggesting that omeprazole increases the risk of HA-AKI even within the normal dosage range.

An unexpected finding of this study was that H2RA might also be associated with an increased risk of HA-AKI in children. Patients treated with H2RA were often used as active controls in previous studies on association between PPI use and kidney disease. Compared with non-users, H2RA users had a 35\% higher risk of HA-AKI in our study. Indeed, the nephrotoxicity of H2RAs has been described in a number of sporadic case reports since 1977 (43,48-50). However, two recent cohort studies in adults failed to find the significant association between H2RAs and AKI $(42,51)$. It has been well established that cimetidine, the most frequently prescribed H2RA (94.6\%) in our study, can block the tubular secretion of creatinine, leading to an increase in the SCr (44). This SCr-increasing effect of cimetidine may result in increased incidence of artifactual "AKI" rather than a real kidney injury. Further studies are needed to dissect and verify the potential association between H2RA use and $\mathrm{AKI}$ in children.

Several limitations in our study should be noted. First, we did not use urine output to identify AKI, as urinary data were not available for most hospitalized children. Second, the cohort only included Chinese children. Whether these results could be generalized to other ethnic population needs further validations. Third, the time sequence between the onset of certain acute comorbidities and use of PPI could not be unambitiously determined, which may lead to inaccurate propensity score models. Fourth, prescription data was only available for around $50 \%$ of patients in our analysis set. Fifth, we did not adjust for the severity of comorbidities in our analysis because we do not have data other than the diagnosis code to index the severity of a disease. This may potentially confound the association between the use of PPI and the risk of AKI. Six, our study was based on hospitalized children. We could neither obtain the prescription information before admission nor assess the long-term effect of PPI. Ignoring the drug use before 
admission would misclassify the patients as no-users and thus attenuate the effect size of PPI towards the null. Lastly, like all other observational studies, despite our attempts to adjust for potential confounders both in traditional approach and with propensity-matching, there is still a very real risk of residual confounding that cannot be controlled in our study.

In summary, PPI use is common and associated with an increased risk of HA-AKI in Chinese children. Our findings call for further risk-and-benefit studies on the use of PPI for prophylaxis purpose, especially in patients at high risk of AKI.

\section{Acknowledgments}

The authors thank the staff and participant centers of the EACH Study for their important contributions.

Funding: This work was supported by the National Key Technology Support Program of China (2015BAI12B07 to FFH), the National Natural Science Foundation of China (Key Program) (81430016 to FFH), the National Natural Science Foundation of China (81770683 and 81970586 to XX), the Science and Technology Planning Project of Guangzhou, China (201607020004 to XX), the Major Scientific and Technological Planning Project of Guangzhou (201504010027 to FFH), and the Major International (Regional) Joint Research Project (81620108003 to FFH).

\section{Footnote}

Reporting Checklist: The authors have completed the STROBE reporting checklist. Available at http://dx.doi. org/10.21037/atm-20-2284

Data Sharing Statement: Available at http://dx.doi. org/10.21037/atm-20-2284

Peer Review File: Available at http://dx.doi.org/10.21037/ atm-20-2284

Conflicts of Interest: All authors have completed the ICMJE uniform disclosure form (available at http://dx.doi. org/10.21037/atm-20-2284). The authors have no conflicts of interest to declare.

Ethical Statement: The authors are accountable for all aspects of the work in ensuring that questions related to the accuracy or integrity of any part of the work are appropriately investigated and resolved. The study was conducted in accordance with the Declaration of Helsinki (as revised in 2013). The study was approved by The Medical Ethics Committee of Nanfang Hospital (No. NFEC-2014098) and individual consent for this retrospective analysis was waived.

Open Access Statement: This is an Open Access article distributed in accordance with the Creative Commons Attribution-NonCommercial-NoDerivs 4.0 International License (CC BY-NC-ND 4.0), which permits the noncommercial replication and distribution of the article with the strict proviso that no changes or edits are made and the original work is properly cited (including links to both the formal publication through the relevant DOI and the license). See: https://creativecommons.org/licenses/by-nc-nd/4.0/.

\section{References}

1. Eckardt KU, Coresh J, Devuyst O, et al. Evolving importance of kidney disease: from subspecialty to global health burden. Lancet 2013;382:158-69.

2. Li PK, Burdmann EA, Mehta RL, et al. Acute kidney injury: global health alert. Kidney Int 2013;83:372-6.

3. Xu X, Nie S, Liu Z, et al. Epidemiology and clinical correlates of AKI in Chinese hospitalized adults. Clin J Am Soc Nephrol 2015;10:1510-8.

4. Susantitaphong P, Cruz DN, Cerda J, et al. World incidence of AKI: a meta-analysis. Clin J Am Soc Nephrol 2013;8:1482-93.

5. Zeng X, McMahon GM, Brunelli SM, et al. Incidence, outcomes, and comparisons across definitions of AKI in hospitalized individuals. Clin J Am Soc Nephrol 2014;9:12-20.

6. Kaddourah A, Basu RK, Bagshaw SM, et al. Epidemiology of Acute Kidney Injury in Critically Ill Children and Young Adults. N Engl J Med 2017;376:11-20.

7. Bellomo R, Kellum JA, Ronco C. Acute kidney injury. Lancet 2012;380:756-66.

8. Uchino S, Kellum JA, Bellomo R, et al. Acute renal failure in critically ill patients: a multinational, multicenter study. JAMA 2005;294:813-8.

9. Mammen C, Al Abbas A, Skippen P, et al. Long-term risk of CKD in children surviving episodes of acute kidney injury in the intensive care unit: a prospective cohort study. Am J Kidney Dis 2012;59:523-30.

10. Sinha R, Nandi M, Tullus K, et al. Ten-year follow-up of children after acute renal failure from a developing 
country. Nephrol Dial Transplant 2009;24:829-33.

11. Askenazi DJ, Feig DI, Graham NM, et al. 3-5 year longitudinal follow-up of pediatric patients after acute renal failure. Kidney Int 2006;69:184-9.

12. Ingelfinger JR, Kalantar-Zadeh K, Schaefer F, et al. Averting the legacy of kidney disease: focus on childhood. Nephrol Dial Transplant 2016;31:327-31.

13. Patzer L. Nephrotoxicity as a cause of acute kidney injury in children. Pediatr Nephrol 2008;23:2159-73.

14. Xu X, Nie S, Zhang A, et al. Acute Kidney Injury among Hospitalized Children in China. Clin J Am Soc Nephrol 2018;13:1791-800.

15. Rosen R, Vandenplas Y, Singendonk M, et al. Pediatric Gastroesophageal Reflux Clinical Practice Guidelines:

Joint Recommendations of the North American Society for Pediatric Gastroenterology, Hepatology, and Nutrition (NASPGHAN) and the European Society for Pediatric Gastroenterology, Hepatology, and Nutrition (ESPGHAN). J Pediatr Gastroenterol Nutr 2018;66:516-54.

16. Koletzko S, Jones NL, Goodman KJ, et al. Evidencebased guidelines from ESPGHAN and NASPGHAN for Helicobacter pylori infection in children. J Pediatr Gastroenterol Nutr 2011;53:230-43.

17. Dellon ES, Gonsalves N, Hirano I, et al. ACG clinical guideline: evidenced based approach to the diagnosis and management of esophageal eosinophilia and eosinophilic esophagi-tis (EoE). Am J Gastroenterol 2013;108:679-92.

18. Barron JJ, Tan H, Spalding J, et al. Proton pump inhibitor utilization patterns in infants. J Pediatr Gastroenterol Nutr 2007;45:421-7.

19. De Bruyne P, Christiaens T, Vander Stichele R, et al. Changes in prescription patterns of acid-suppressant medications by Belgian pediatricians: analysis of the national database, [1997-2009]. J Pediatr Gastroenterol Nutr 2014;58:220-5.

20. Forgacs I, Loganayagam A. Overprescribing proton pump inhibitors. BMJ 2008;336:2-3.

21. Orenstein SR, Hassall E, Furmaga-Jablonska W, et al. Multicenter, double-blind, randomized, placebocontrolled trial assessing the efficacy and safety of proton pump inhibitor lansoprazole in infants with symptoms of gastroesophageal reflux disease. J Pediatr 2009;154:514-520.e4.

22. Moore DJ, Tao BS, Lines DR, et al. Double-blind placebo-controlled trial of omeprazole in irritable infants with gastroesophageal reflux. J Pediatr 2003;143:219-23.

23. Blank ML, Parkin L, Paul C, et al. A nationwide nested case-control study indicates an increased risk of acute interstitial nephritis with proton pump inhibitor use. Kidney Int 2014;86:837-44.

24. Klepser DG, Collier DS, Cochran GL. Proton pump inhibitors and acute kidney injury: a nested case-control study. BMC Nephrol 2013;14:150.

25. Sierra F, Suarez M, Rey M, et al. Systematic review: Proton pump inhibitor-associated acute interstitial nephritis. Aliment Pharmacol Ther 2007;26:545-53.

26. Antoniou T, Macdonald EM, Hollands S, et al. Proton pump inhibitors and the risk of acute kidney injury in older patients: a population-based cohort study. CMAJ Open 2015;3:E166-71.

27. Nochaiwong S, Ruengorn C, Awiphan R, et al. The association between proton pump inhibitor use and the risk of adverse kidney outcomes: a systematic review and meta-analysis. Nephrol Dial Transplant 2018;33:331-42.

28. Xu X, Nie S, Zhang A, et al. A New criterion for pediatric AKI based on the reference change value of serum creatinine. J Am Soc Nephrol 2018;29:2432-42.

29. Kidney Disease: Improving Global Outcomes (KDIGO) Acute Kidney Injury Work Group. KDIGO Clinical Practice Guideline for Acute Kidney Injury. Kidney Inter Suppl 2012;2:1-138.

30. Austin PC. A Tutorial and case study in propensity score analysis: an application to estimating the effect of inHospital smoking cessation counseling on mortality. Multivariate Behav Res 2011;46:119-51.

31. R Core Team. R: A language and environment for statistical computing. R Foundation for Statistical Computing, Vienna, Austria. 2019. Available online: http://www.R-project.org/

32. Yang $\mathrm{X}$, Chen $\mathrm{C}$, Teng $\mathrm{S}$, et al. Urinary matrix metalloproteinase-7 predicts severe AKI and poor outcomes after cardiac surgery. J Am Soc Nephrol 2017;28:3373-82.

33. Johansen ME, Huerta TR, Richardson CR. National use of proton pump inhibitors from 2007 to 2011. JAMA Intern Med 2014;174:1856-8.

34. Ahrens D, Chenot JF, Behrens G, et al. Appropriateness of treatment recommendations for PPI in hospital discharge letters. Eur J Clin Pharmacol 2010;66:1265-71.

35. Delcher A, Hily S, Boureau AS, et al. Multimorbidities and overprescription of proton pump inhibitors in older patients. PLoS One 2015;10:e0141779.

36. Wilhelm SM, RjaterRG, Kale-Pradhan PB. Perils and pitfalls of long-term effects of proton pump inhibitors. Expert Rev Clin Pharmacol 2013;6:443-51. 
37. Klatte DCF, Gasparini A, Xu H, et al. Association between proton pump inhibitor use and risk of progression of chronic kidney disease. Gastroenterology 2017;153:702-10.

38. Slaughter JL, Stenger MR, Reagan PB, et al. Neonatal histamine-2 receptor antagonist and proton pump inhibitor treatment at United States children's hospitals. J Pediatr 2016;174:63-70.e3.

39. Ward RM, Kearns GL. Proton pump inhibitors in pediatrics: mechanism of action, pharmacokinetics, pharmacogenetics, and pharmacodynamics. Paediatr Drugs 2013;15:119-31.

40. Alhazzani W, Alshamsi F, Belley-Cote E, et al. Efficacy and safety of stress ulcer prophylaxis in critically ill patients: a network meta-analysis of randomized trials. Intensive Care Med 2018;44:1-11.

41. Praga M, González E. Acute interstitial nephritis. Kidney Int 2010;77:956-61.

42. Lazarus B, Chen Y, Wilson FP, et al. Proton pump inhibitor use and the risk of chronic kidney disease. JAMA Intern Med 2016;176:238-46.

43. Fisher AA, Le Couteur DG. Nephrotoxicity and hepatotoxicity of histamine $\mathrm{H} 2$ receptor antagonists. Drug
Saf 2001;24:39-57.

44. Lepist EI, Zhang X, Hao J, et al. Contribution of the organic anion transporter OAT2 to the renal active tubular secretion of creatinine and mechanism for serum creatinine elevations caused by cobicistat. Kidney Int 2014;86:350-7.

45. Perazella MA, Markowitz GS. Drug-induced acute interstitial nephritis. Nat Rev Nephrol 2010;6:461-70.

46. Moledina DG, Perazella MA. PPIs and kidney disease: from AIN to CKD. J Nephrol 2016;29:611-6.

47. Berney-Meyer L, Hung N, Slatter T, et al. Omeprazoleinduced acute interstitial nephritis: a possible Th1-Th17mediated injury? Nephrology (Carlton) 2014;19:359-65.

48. Grave W, Nadorp JN, Rutten JJ. Cimetidine and renal failure. Lancet 1977;2:719-20.

49. Rudnick MR, Bastl CP, Elfenbein IB, et al. Cimetidineinduced acute renal failure. Ann Intern Med 1982;96:180-2.

50. Hirayama K, Hanatsuka K, Ikeuchi T, et al. Famotidineinduced acute interstitial nephritis. Nephrol Dial Transplant 1998;13:2636-8.

51. Lee J, Mark RG, Celi LA, et al. Proton pump inhibitors are not associated with acute kidney injury in critical Illness. J Clin Pharmacol 2016;56:1500-6.
Cite this article as: Li Y, Xiong M, Yang M, Wang L, Nie S, Liu D, Pi M, Zhang A, Mao J, Liu HP, Xia H, Xu H, Liu Z, Feng S, Zhou W, Liu X, Yang Y, Tao Y, Feng Y, Chen C, Wang M, Zha Y, Feng JH, Li Q, Ge S, Chen J, He Y, Teng S, Hao C, Liu BC, Tang Y, He W, Hou FF, Xu X. Proton pump inhibitors and the risk of hospital-acquired acute kidney injury in children. Ann Transl Med 2020;8(21):1438. doi: 10.21037/atm20-2284 